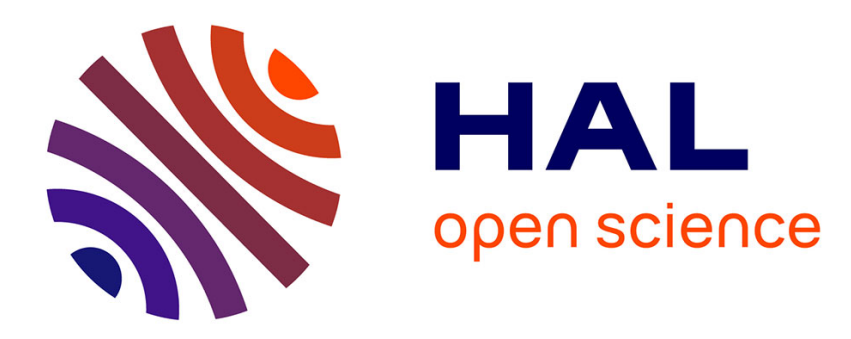

\title{
Comparison of Numerical Differentiation Techniques for Aircraft Identification
}

Mathieu Brunot

\section{To cite this version:}

Mathieu Brunot. Comparison of Numerical Differentiation Techniques for Aircraft Identification. Journal of Aerospace Engineering, 2019, 32 (5), pp.1-5. 10.1061/(ASCE)AS.1943-5525.0001003 . hal02313837

\section{HAL Id: hal-02313837 \\ https://hal.science/hal-02313837}

Submitted on 11 Oct 2019

HAL is a multi-disciplinary open access archive for the deposit and dissemination of scientific research documents, whether they are published or not. The documents may come from teaching and research institutions in France or abroad, or from public or private research centers.
L'archive ouverte pluridisciplinaire HAL, est destinée au dépôt et à la diffusion de documents scientifiques de niveau recherche, publiés ou non, émanant des établissements d'enseignement et de recherche français ou étrangers, des laboratoires publics ou privés. 


\title{
Comparison of Numerical Differentiation Techniques for Aircraft Identification
}

\author{
Mathieu Brunot ${ }^{1}$ \\ ${ }^{1}$ Research Engineer, PhD, DTIS, ONERA, F-31055 Toulouse, FRANCE. Email: \\ mathieu.brunot@onera.fr
}

\begin{abstract}
External disturbance and measurement noise during flight tests inevitably degrade the identification of the aircraft aerodynamic models. Traditional approaches, however, need to differentiate the measured signals to build the identification models, which results in a dedicated pre-processing to avoid noise amplification. The aim of this technical note is to assess the influence of four derivative estimation techniques on the parameter estimation of an aircraft aerodynamic model. Amongst the four studied techniques, two come from the field of robot identification. The other two techniques are the standard one in aircraft identification based on the Savitzky-Golay algorithm and a suggested one based on wavelet denoising coupled with finite differences. The two techniques coming from robot identification are the usual one relying on a low-pass filter applied in both forward and backward directions and a recently suggested method based on a Kalman filter with a first order random walk model. The comparison simulation results illustrate that the first robot differentiation strategy not only performs well in providing accurate stability and control derivatives even in the presence of colored disturbance, but also is competitive with respect to the standard method in aircraft identification.
\end{abstract}

\section{INTRODUCTION}

The well-known equation-error approach for aircraft identification requires the estimation of derivatives from noisy measurement signals. That allows constructing an identification model linear with respect to the parameters, which can thus be estimated with a linear regression technique. 
The goal of this technical note is to suggest and evaluate techniques to estimate the derivatives used in robot identification. The usual approach in aircraft identification, based on polynomial interpolation, described in (Morelli 2006; Klein and Morelli 2006) is considered as well as a method combining finite differences and wavelet denoising. The two suggested techniques from robot identification are a combination of finite differences with a pre-filter and a Kalman filter with a random walk model. The former is the standard technique for industrial robot identification (Gautier 1997) and the latter has recently proven to be a viable alternative (Brunot et al. 2018).

For this note, the author considers the reduced order model of the Short Period Pitch Oscillation (SPPO) to be concise. Eq. (1) gives the linearized state equation corresponding to this mode and (2) provides the measurement model, where $v_{\alpha}, v_{q}$ and $v_{a_{z}}$ are measurement noises assumed to be white. For the reader unfamiliar with the flight dynamics notations, an appendix summarizes those used in this brief.

$$
\begin{aligned}
{\left[\begin{array}{c}
\dot{\alpha} \\
\dot{q}
\end{array}\right] } & =\left[\begin{array}{cc}
-\frac{\bar{q} S}{m V} C_{L \alpha} & 1-\frac{\bar{q} S}{m V} \frac{\bar{c}}{2 V} C_{L q} \\
\frac{\bar{q} S \bar{c}}{I_{y y}} C_{m \alpha} & \frac{\bar{q} S \bar{c}}{I_{y y}} \frac{\bar{c}}{2 V} C_{m q}
\end{array}\right]\left[\begin{array}{l}
\alpha \\
q
\end{array}\right]+\left[\begin{array}{c}
-\frac{\bar{q} S}{m V} C_{L \delta_{e}} \\
\frac{\bar{q} S \bar{c}}{I_{y y}} C_{m \delta_{e}}
\end{array}\right] \delta_{e} \\
{\left[\begin{array}{c}
\alpha_{m} \\
q_{m} \\
a_{z_{m}}
\end{array}\right] } & =\left[\begin{array}{cc}
1 & 0 \\
0 & 1 \\
-\frac{\bar{q} S}{m g} C_{L \alpha} & -\frac{\bar{q} S}{m g} \frac{\bar{c}}{2 V} C_{L q}
\end{array}\right]\left[\begin{array}{c}
\alpha \\
q
\end{array}\right]+\left[\begin{array}{c}
0 \\
0 \\
-\frac{\bar{q} S}{m g} C_{L \delta_{e}}
\end{array}\right] \delta_{e}+\left[\begin{array}{c}
v_{\alpha} \\
v_{q} \\
v_{a_{z}}
\end{array}\right]
\end{aligned}
$$

From those equations, a formulation linear with respect to the parameters to be estimated, referred to as the identification model, can be derived:

$$
\boldsymbol{y}=\left[\begin{array}{lll}
\boldsymbol{y}_{1}^{T} & \boldsymbol{y}_{2}^{T} & \boldsymbol{y}_{3}^{T}
\end{array}\right]^{T}=\boldsymbol{X} \boldsymbol{\theta}+\boldsymbol{v}
$$

where

$$
\begin{aligned}
& \text { - } \boldsymbol{y}_{i}=\operatorname{vec}\left(y_{i}(t)\right)=\left[\begin{array}{lll}
y_{i}\left(t_{1}\right) & \cdots & y_{i}\left(t_{N}\right)
\end{array}\right]^{T} \text { is a }(N \times 1) \text { vector of } y_{i}, \text { with } y_{1}=\dot{\alpha}-q_{m}, y_{2}=\dot{q} \\
& \text { and } y_{3}=a_{z_{m}}
\end{aligned}
$$


- $\boldsymbol{\theta}=\left[\begin{array}{llllll}C_{L \alpha} & C_{L q} & C_{L \delta_{e}} & C_{m \alpha} & C_{m q} & C_{m \delta_{e}}\end{array}\right]^{T}$ is a $(6 \times 1)$ vector of unknown parameters;

- $\boldsymbol{v}=\left[\begin{array}{lll}v_{1}^{T} & v_{2}^{T} & v_{3}^{T}\end{array}\right]^{T}$ is a $(3 N \times 1)$ vector of error terms $v_{i}$, with $v_{i}=\operatorname{vec}\left(v_{i}(t)\right)=$ $\left[\begin{array}{lll}v_{i}\left(t_{1}\right) & \cdots & v_{i}\left(t_{N}\right)\end{array}\right]^{T}$

- $\boldsymbol{X}=\left[\begin{array}{cccccc}\frac{1}{m V} \boldsymbol{X}_{\alpha} & \frac{1}{m V} \boldsymbol{X}_{q} & \frac{1}{m V} \boldsymbol{X}_{\delta_{e}} & \mathbf{0}_{N} & \mathbf{0}_{N} & \mathbf{0}_{N} \\ \mathbf{0}_{N} & \mathbf{0}_{N} & \mathbf{0}_{N} & -\frac{\bar{c}}{I_{y y}} \boldsymbol{X}_{\alpha} & -\frac{\bar{c}}{I_{y y}} \boldsymbol{X}_{q} & -\frac{\bar{c}}{I_{y y}} \boldsymbol{X}_{\delta_{e}} \\ \frac{1}{m g} \boldsymbol{X}_{\alpha} & \frac{1}{m g} \boldsymbol{X}_{q} & \frac{1}{m g} \boldsymbol{X}_{\delta_{e}} & \mathbf{0}_{N} & \mathbf{0}_{N} & \mathbf{0}_{N}\end{array}\right]$ is the $(3 N \times 6)$ observation matrix built with $\boldsymbol{X}_{\alpha}=\operatorname{vec}(-\bar{q} S \alpha(t)), \boldsymbol{X}_{q}=\operatorname{vec}\left(-\bar{q} S \frac{\bar{c}}{2 V} q(t)\right)$ and $\boldsymbol{X}_{\delta_{e}}=\operatorname{vec}\left(-\bar{q} S \delta_{e}(t)\right)$.

Based on (3), the parameters can be estimated straightforwardly with the Least-Squares (LS) method (Klein and Morelli 2006):

$$
\widehat{\boldsymbol{\theta}}=\left(\boldsymbol{X}^{T} \boldsymbol{X}\right)^{-1} \boldsymbol{X}^{T} \boldsymbol{y}
$$

With (3), it appears the need to evaluate the derivatives $\dot{\alpha}$ and $\dot{q}$ from the measurements coming from (2). Angular accelerations are usually not measured, or else with poor quality. The pre-processing step must be accurately done in order to limit the distortions of the signals. That disqualifies the use of the finite difference formulas without any filtering, because it would amplify high frequency noise components: see e.g. (Wood 1982).

Two important aspects of this pre-processing must be noted. Firstly, in this note, only off-line identification is considered; i.e. the whole time series are available. Consequently, the possibility of using future data for the filtering is left opened. Secondly, the objective is to pre-filter only the signals $\alpha_{m}, q_{m}$ and $a_{z_{m}}$. Therefore, the pre-processing step cannot introduce any lag in the time series in order to use the derivatives along with unprocessed signals, like the elevator input for instance. In the following section, the pre-processing techniques compared in this note are summarized.

\section{CONSIDERED NUMERICAL DIFFERENTIATION TECHNIQUES}




\section{Filtered Finite Differences Technique}

To avoid the noise amplification by Finite Differences (FD), the basic idea is to filter the data before, as described in (Gautier 1997). The measured signal $x_{m}$ is firstly filtered to obtain $\widehat{x}$. From this filtered signal, the derivative can be calculated with FD. The filter type and its cut-off frequency, $f_{c}$, must be selected such as $(\widehat{x}, \widehat{\dot{x}}) \approx(x, \dot{x})$ in the range $\left[0, f_{c}\right]$. In robot identification, the filter is usually a Butterworth one and it is applied in both forward and reverse directions to avoid lag introduction. For robots, the rule of thumb for the cut-off frequency is $f_{c} \geq 5 f_{d y n}$, where $f_{d y n}$ is the natural frequency of the highest mode of the system.

At first glance, the use of the Butterworth filter presents two drawbacks. Firstly, it does not separate the noise from the signal if the Fourier spectra overlap. Secondly, it requires a manual selection of the frequency range based on the practitioner knowledge. An alternative to avoid such limitations is the use of wavelets for signal denoising. In this method, the measured signal is decomposed into a set of generating wavelets functions. A threshold is used to delete the wavelet coefficients where the signal is smooth and keep the coefficients which are large enough. In this brief, the author uses the denoising function ThreshWave from the WaveLab Toolbox prior to FD: see e.g. (Donoho and Johnstone 1995). The Toolbox can be downloaded via http://statweb.stanford.edu/ wavelab/.

\section{Polynomial Least-Squares Approximation}

The polynomial interpolation over a sliding window is a solution for local smoothing of noisy time series, but it can also provide an estimate of the derivative. The principle is to fit a low-degree polynomial over successive sub-sets of adjacent data points. From the estimated local polynomials, the local derivative values can be estimated. The user must thus set the size of the window and the order of the polynomial. That method is often referred to as the Savitzky-Golay algorithm. For this brief, the implementation of the SIDPAC (System IDentication Programs for AirCraft) (Morelli 2002) is employed. This Toolbox can be downloaded via https://software.nasa.gov/software/LAR16100-1. The deriv routine indeed provides an effective implementation of this method with default settings that gave good results in many practical cases of flight test data analysis. 


\section{Time-Varying Parameter Estimation}

An automatic alternative method that does not require a priori knowledge of the system bandwidth is based on a combination of the Kalman Filter and Fixed Interval Smoother (KF-FIS). This is described in chapter 4 of (Young 2011) and has been applied successfully to robot identification (Brunot et al. 2018). This same approach can be used in the present context by modeling the signal $x$ as a simple Integrated Random Walk (IRW) process described by a simple state equation of the form,

$$
\begin{aligned}
{\left[\begin{array}{c}
x\left(t_{i}\right) \\
\dot{x}\left(t_{i}\right)
\end{array}\right] } & =\left[\begin{array}{cc}
1 & \Delta t \\
0 & 1
\end{array}\right]\left[\begin{array}{l}
x\left(t_{i-1}\right) \\
\dot{x}\left(t_{i-1}\right)
\end{array}\right]+\left[\begin{array}{l}
0 \\
1
\end{array}\right] \varpi\left(t_{i-1}\right), \\
x_{m}\left(t_{i}\right) & =x\left(t_{i}\right)+\xi\left(t_{i}\right),
\end{aligned}
$$

where $\varpi$ and $\xi$ are, respectively, the process and measurement noise inputs; $x$ and $\dot{x}$ are the states to be estimated; $\Delta t$ is the fixed sampling period. The variances of the noises are regrouped in a single hyper-parameter, the Noise Variance Ratio (NVR):

$$
n v r=\frac{\operatorname{var}(\varpi)}{\operatorname{var}(\xi)}
$$

This NVR is estimated thanks to a maximum likelihood optimization. The resulting KF-FIS algorithm yields the estimates $\widehat{x}$ and $\widehat{\dot{x}}$ that are required to construct the observation matrix. This Integrated Random Walk SMoothing (IRWSM) algorithm is coded as the routine irwsm in the CAPTAIN Toolbox, which can be downloaded via http://captaintoolbox.co.uk. It should be stressed that, thanks to the maximum likelihood optimization, the practitioner does not have to provide any a priori knowledge.

Table 2 summarizes the considered methods for the estimation of the derivatives, which are divided in two steps. The first step consists in filtering the noisy signal without lag introduction. The second step focuses on obtaining the derivative signal, which is relatively straightforward for the polynomial and IRWSM methods. 


\section{RESULTS}

\section{Influence of the Sampling}

For the numerical evaluation of the techniques, the T-2 aircraft model used in (Grauer and Morelli 2015) is considered. The parameters and the noise levels are taken equal to the estimated values for Flight 15 in the previous reference. Still according to that reference, the aircraft is excited with a $10 \mathrm{~s}$ orthogonal phase-optimized multisine input with frequencies located between $0.2 \mathrm{~Hz}$ and $2.2 \mathrm{~Hz}$ whereas the data are sampled at $50 \mathrm{~Hz}$ for analysis and modeling. In practice, the mkmsswp function of the SIDPAC is used to generate the elevator deflection. In addition, $1 \mathrm{~s}$ and $2 \mathrm{~s}$ of zero excitation are added respectively before and after the perturbation (Klein and Morelli 2006).

Regarding the FD, we use a $4^{\text {th }}$ order Butterworth filter with a cut-off frequency $f_{c}=2 f_{d y n}=$ 4.4 Hz. For the wavelet denoising, ThreshWave is used with the default parameters and hard thresholding. As explained in Section 2, deriv is used with default settings for the polynomial approximation. Concerning the IRWSM technique, the routines of the CAPTAIN are self-contained.

In a first time, the influence of the sampling time is investigated. To avoid any combination of two problems, the identification is performed without noise. In this case, the IRWSM approach is not considered since it would mean the $n v r$ is infinite. Table 3 summarizes the LS estimates and their relative errors, defined by (7) where $x_{s}$ is the noise-free/true vector and $\widehat{x}$ is its estimate. At the regular sampling frequency $\left(F_{S}=50 \mathrm{~Hz}\right)$, the estimates appear to be slightly biased whereas there is no noise. Those discrepancies come from the error in the derivative estimation; see Table 4 that gives the relative errors of the derivatives. This preliminary test shows that the estimation is very sensitive to any distortion in the derivative estimation and that it is preferable to have the largest sampling frequency possible. Furthermore, it illustrates the bottom line of what can be observed with noisy data.

$$
\operatorname{RelErr}(x)=\frac{\left\|x_{s}-\widehat{x}\right\|}{\left\|x_{s}\right\|}
$$




\section{Influence of the White Noise}

In this part, the techniques are evaluated with added white noises on the outputs. According to (Grauer and Morelli 2015), the measurement noise standard deviations are $\sigma_{v_{\alpha}}=0.232 \mathrm{deg}$, $\sigma_{v_{q}}=0.274 \mathrm{deg} / \mathrm{s}$ and $\sigma_{v_{a_{z}}}=0.045 \mathrm{~g}$ in the case of severe turbulence. The sampling frequency is $50 \mathrm{~Hz}$ and 500 Monte Carlo Simulations (MCS) with independent noise sequences are run.

Table 5 gives the mean LS estimates, their standard deviations and their relative errors. The mean relative errors of the derivatives can be found in Table 4. It appears that the IRWSM technique has more difficulties to provide accurate estimates compared with other techniques whereas the estimation of the derivatives is not especially biased. That can be explained by the error on the estimation of $\dot{q}$ that leads to a biased $C_{m q}$, which has a large influence in the computation of the relative error of $\theta$. Such an estimation error can be attributed to the sampling frequency. The random walk model of the IRWSM technique indeed assumes that the states are slowly varying. In the present case, the system's dynamics should be too close to the sampling rate. That was confirmed by a test with $F_{s}=500 \mathrm{~Hz}$. All the results are not shown for clarity but the relative error fell to $2.32 \%$ for the IRWSM estimated parameters. The polynomial technique provides a better estimation of the derivatives than the Wavelet technique. However, its LS estimates are less accurate than those of the Butterworth and Wavelet techniques. The Wavelet technique provides appropriate LS estimates. Nonetheless, its large relative errors for the derivatives reflect non-negligible oscillations in the time history: see Fig. 1 for an illustration. For comparison, the corresponding Butterworth estimated derivative is given in Fig. 2 to illustrate an appropriate estimation of $\dot{\alpha}$. Finally, the Butterworth technique seems to be the most effective by looking at the relative errors of the derivatives and the final LS estimates.

The careful reader would have noticed that the relative errors are larger for the estimation of $\dot{\alpha}$ whereas the related parameters $\left(C_{L \alpha}, C_{L q}\right.$ and $\left.C_{L \delta_{e}}\right)$ do not seem too impacted during the LS estimation. That is likely due to the fact those parameters are also estimated through $a_{z_{m}}$ which is not deteriorated by a differentiation. An important lesson to be noted is that, with this model, simple rules cannot be inferred to link the quality of the derivatives estimation and the one of the 
aerodynamic parameters.

\section{Influence of the Colored Noise}

The techniques are now evaluated with colored noise. According to (Morelli and Cunningham 2012), it is adequate to confine $80 \%$ of the noise power in the input frequency range. Consequently, a Finite Impulse Response filter is designed with an unitary gain below $4 \mathrm{~Hz}$ to colored the white noise used in the previous part.

The LS results are presented in Table 6 and the mean relative errors of the derivatives estimation are given in Table 4. First of all, some of the IRWSM estimates were removed due to their nonfeasibility ( $\boldsymbol{X}$ rank deficient or $C_{m q}>0$ ). Despite this, the IRWSM estimated parameters are not satisfactory. That is likely due to the bad estimation of $\dot{q}$. Besides the limitation seen in the previous part, the colored noise violates the assumptions of the Kalman filter. The Butterworth and Wavelet techniques equivalently perform with respect to the LS and derivatives estimation. The polynomial technique results are also far from inadmissible. Except the IRWSM technique, the other ones seem robust to the colored aspect of the noise. Their results are indeed close to the bottom line found in Table 3. In fact, due to the filter design, the overall energy of the noise is less important than in the white noise case.

\section{CONCLUSION}

In this brief paper, several numerical differentiation techniques used in robot identification have been compared on an aircraft parameter estimation problem. The least-squares estimation method indeed requires the knowledge of unmeasured signals that must be reconstructed via a numerical differentiation technique. All the considered methods rely on freely available Toolboxes and are tractable in a few seconds on a standard computer. Monte Carlo simulations were run and have shown that:

- Sample rate is critical to have a derivative estimation accurate enough;

- IRWSM technique for estimating system parameters does not seem appropriate due to a sampling frequency too close to the system's dynamics; 


$$
\begin{aligned}
& a_{z}=\text { normal acceleration }\left[m \cdot s^{-2}\right] \\
& C_{L}=\text { lift force coefficient } \\
& C_{m}=\text { pitching moment coefficient } \\
& \bar{c}=\text { mean aerodynamic chord }[m] \\
& g=\text { gravitational acceleration }\left[m . s^{-2}\right] \\
& I_{y y}=\text { pitch moment of inertia }\left[\mathrm{kg} \cdot \mathrm{m}^{2}\right] \\
& m=\operatorname{mass}[\mathrm{kg}] \\
& N=\text { number of sample } \\
& q=\text { body-axis pitch rate }[\mathrm{rad} / \mathrm{s}] \\
& \bar{q}=\text { dynamic pressure }\left[k g \cdot m^{-1} \cdot s^{-2}\right] \\
& S=\text { wing reference area }\left[\mathrm{m}^{2}\right] \\
& t=\text { time }[s] \\
& V=\text { true airspeed }\left[\mathrm{m} . \mathrm{s}^{-1}\right] \\
& \alpha=\text { angle of attack }[\mathrm{rad}] \\
& \delta_{e}=\text { elevator deflection }[\mathrm{rad}]
\end{aligned}
$$

Subscripts

$$
m=\text { measured value }
$$


Superscripts

$$
\begin{aligned}
-1 & =\text { inverse } \\
T & =\text { transpose } \\
- & =\text { estimated value }
\end{aligned}
$$

\section{REFERENCES}

Brunot, M., Janot, A., Young, P. C., and Carrillo, F. (2018). “An instrumental variable method for robot identification based on time variable parameter estimation [special issue]." Kybernetika, 54(1), 202-220.

Donoho, D. L. and Johnstone, I. M. (1995). “Adapting to unknown smoothness via wavelet shrinkage." Journal of the american statistical association, 90(432), 1200-1224.

Gautier, M. (1997). "Dynamic identification of robots with power model.” Robotics and Automation, 1997. Proceedings., 1997 IEEE International Conference on, Vol. 3, IEEE, 1922-1927.

Grauer, J. A. and Morelli, E. A. (2015). "A new formulation of the filter-error method for aerodynamic parameter estimation in turbulence." AIAA Atmospheric Flight Mechanics Conference, 2704.

Klein, V. and Morelli, E. A. (2006). Aircraft system identification: theory and practice. American Institute of Aeronautics and Astronautics Reston, Va, USA.

Morelli, E. (2002). "System identification programs for aircraft (sidpac)." AIAA Atmospheric Flight Mechanics Conference and Exhibit, 4704.

Morelli, E. (2006). "Practical aspects of the equation-error method for aircraft parameter estimation." AIAA Atmospheric Flight Mechanics Conference and Exhibit, 6144.

Morelli, E. and Cunningham, K. (2012). "Aircraft dynamic modeling in turbulence." AIAA Atmospheric Flight Mechanics Conference, 4650.

Wood, G. A. (1982). "Data smoothing and differentiation procedures in biomechanics." Exercise and sport sciences reviews, 10(1), 308-362. 
Young, P. C. (2011). Recursive estimation and time-series analysis: an introduction for the student and practitioner. Springer Berlin Heidelberg, 2nd edition. 


\section{List of Tables}

2 Summary table of the considered methods . . . . . . . . . . . . 13

$3 \widehat{\theta}-$ Noise-free case . . . . . . . . . . . . . . . . . 14

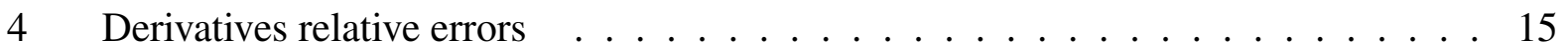

5 White-noise case - 500 MCS - Mean estimated parameters and standard deviations 16

6 Colored-noise case - 500 MCS - Mean estimated parameters and standard deviations 17 
TABLE 2. Summary table of the considered methods

\begin{tabular}{c|cccc}
\hline Filtering & Butterworth & Wavelet & Polynomial & IRWSM \\
\hline Dutterworth filter & $\begin{array}{c}\text { Wavelet } \\
\text { denoising }\end{array}$ & $\begin{array}{c}\text { Polynomial } \\
\text { interpolation }\end{array}$ & $\begin{array}{c}\text { Kalman filter \& } \\
\text { Fixed interval } \\
\text { smoother }\end{array}$ \\
\hline & $\begin{array}{c}\text { Finite } \\
\text { Differences }\end{array}$ & $\begin{array}{c}\text { Finite } \\
\text { Differences }\end{array}$ & $\begin{array}{c}\text { Estimated } \\
\text { derivative of the } \\
\text { polynomial }\end{array}$ & $\begin{array}{c}\text { Estimated state } \\
\text { derivative }\end{array}$ \\
\hline
\end{tabular}


TABLE 3. $\widehat{\theta}$ - Noise-free case

\begin{tabular}{cccccccc}
\hline & & \multicolumn{3}{c}{$F_{s}=50 \mathrm{~Hz}$} & \multicolumn{3}{c}{$F_{s}=500 \mathrm{~Hz}$} \\
$\theta$ & True Value & Butterworth & Wavelet & Polynomial & Butterworth & Wavelet & Polynomial \\
\hline$C_{L \alpha}$ & 3.828 & 3.827 & 3.827 & 3.825 & 3.828 & 3.828 & 3.828 \\
$C_{L q}$ & 16.39 & 16.45 & 16.51 & 16.78 & 16.38 & 16.39 & 16.39 \\
$C_{L \delta_{e}}$ & 0.125 & 0.125 & 0.125 & 0.125 & 0.125 & 0.125 & 0.125 \\
$C_{m \alpha}$ & -1.437 & -1.429 & -1.430 & -1.416 & -1.437 & -1.437 & -1.437 \\
$C_{m q}$ & -44.76 & -44.09 & -44.19 & -42.98 & -44.61 & -44.75 & -44.74 \\
$C_{m \delta_{e}}$ & -1.722 & -1.699 & -1.702 & -1.666 & -1.718 & -1.722 & -1.721 \\
\hline & $\operatorname{RelErr}(\widehat{\theta})$ & $1.41 \%$ & $1.22 \%$ & $3.82 \%$ & $0.31 \%$ & $0.01 \%$ & $0.04 \%$ \\
\hline
\end{tabular}


TABLE 4. Derivatives relative errors

\begin{tabular}{cccccc}
\hline & Technique & Noise-free $(50 \mathrm{~Hz})$ & Noise-free $(500 \mathrm{~Hz})$ & White noise & Colored noise \\
\hline & Butterworth & $0.70 \%$ & $0.49 \%$ & $22.7 \%$ & $17.5 \%$ \\
$\dot{\alpha}$ & Wavelet & $0.53 \%$ & $0.01 \%$ & $98.3 \%$ & $19.9 \%$ \\
& Polynomial & $1.78 \%$ & $0.02 \%$ & $60.8 \%$ & $18.2 \%$ \\
& IRWSM & - & - & $21.4 \%$ & $23.4 \%$ \\
\hline \multirow{4}{*}{$\dot{q}$} & Butterworth & $2.05 \%$ & $1.94 \%$ & $3.76 \%$ & $3.18 \%$ \\
Wavelet & $1.11 \%$ & $0.02 \%$ & $14.7 \%$ & $2.98 \%$ \\
& Polynomial & $2.83 \%$ & $0.04 \%$ & $8.90 \%$ & $3.97 \%$ \\
& IRWSM & - & - & $15.7 \%$ & $189 \%$ \\
\hline
\end{tabular}


TABLE 5. White-noise case - 500 MCS - Mean estimated parameters and standard deviations

\begin{tabular}{cccccc}
\hline$\theta$ & True Value & Butterworth & Wavelet & Polynomial & IRWSM \\
\hline$C_{L \alpha}$ & 3.828 & $3.796(0.054)$ & $3.711(0.055)$ & $3.634(0.051)$ & $3.824(0.054)$ \\
$C_{L q}$ & 16.39 & $17.24(3.722)$ & $18.56(3.733)$ & $20.85(3.648)$ & $18.14(3.679)$ \\
$C_{L \delta_{e}}$ & 0.125 & $0.129(0.062)$ & $0.134(0.062)$ & $0.145(0.061)$ & $0.148(0.062)$ \\
$C_{m \alpha}$ & -1.437 & $-1.418(0.013)$ & $-1.388(0.014)$ & $-1.346(0.012)$ & $-1.396(0.013)$ \\
$C_{m q}$ & -44.76 & $-44.30(0.969)$ & $-45.11(0.978)$ & $-44.27(0.919)$ & $-54.54(0.951)$ \\
$C_{m \delta_{e}}$ & -1.722 & $-1.700(0.017)$ & $-1.711(0.016)$ & $-1.672(0.016)$ & $-1.758(0.016)$ \\
\hline \multicolumn{7}{r}{} & $\operatorname{RelErr}(\widehat{\theta})$ & $2.01 \%$ & $4.60 \%$ & $9.39 \%$ & $20.8 \%$ \\
\hline
\end{tabular}


TABLE 6. Colored-noise case - 500 MCS - Mean estimated parameters and standard deviations

\begin{tabular}{|c|c|c|c|c|c|}
\hline$\theta$ & True Value & Butterworth & Wavelet & Polynomial & IRWSM* \\
\hline$C_{L \alpha}$ & 3.828 & $3.801(0.055)$ & $3.799(0.055)$ & $3.797(0.055)$ & $3.828(0.055)$ \\
\hline$C_{L q}$ & 16.39 & $17.11(3.750)$ & 17.16 (3.749) & 17.44 (3.747) & $18.86(3.696)$ \\
\hline$C_{L \delta_{e}}$ & 0.125 & $0.126(0.064)$ & $0.126(0.064)$ & $0.126(0.064)$ & $0.166(0.063)$ \\
\hline$C_{m \alpha}$ & -1.437 & $-1.420(0.013)$ & $-1.420(0.013)$ & $-1.406(0.013)$ & $-1.406(0.194)$ \\
\hline$C_{m q}$ & -44.76 & $-44.28(0.965)$ & $-44.39(0.964)$ & $-43.18(0.955)$ & $-49.95(56.53)$ \\
\hline \multirow[t]{2}{*}{$C_{m \delta_{e}}$} & -1.722 & $-1.700(0.016)$ & $-1.703(0.016)$ & $-1.667(0.016)$ & $-1.728(0.238)$ \\
\hline & $\operatorname{RelErr}(\widehat{\theta})$ & $1.80 \%$ & $1.78 \%$ & $3.96 \%$ & $12.0 \%$ \\
\hline
\end{tabular}




\section{List of Figures}

1 Example of $\dot{\alpha}$ estimation - White-noise - Wavelet (dashed) and true noise-free signal (solid) . . . . . . . . . . . . . . . . . . . 19

2 Example of $\dot{\alpha}$ estimation - White-noise - Butterworth (dashed) and true noise-free signal (solid) . . . . . . . . . . . . . . . . . . . 20 


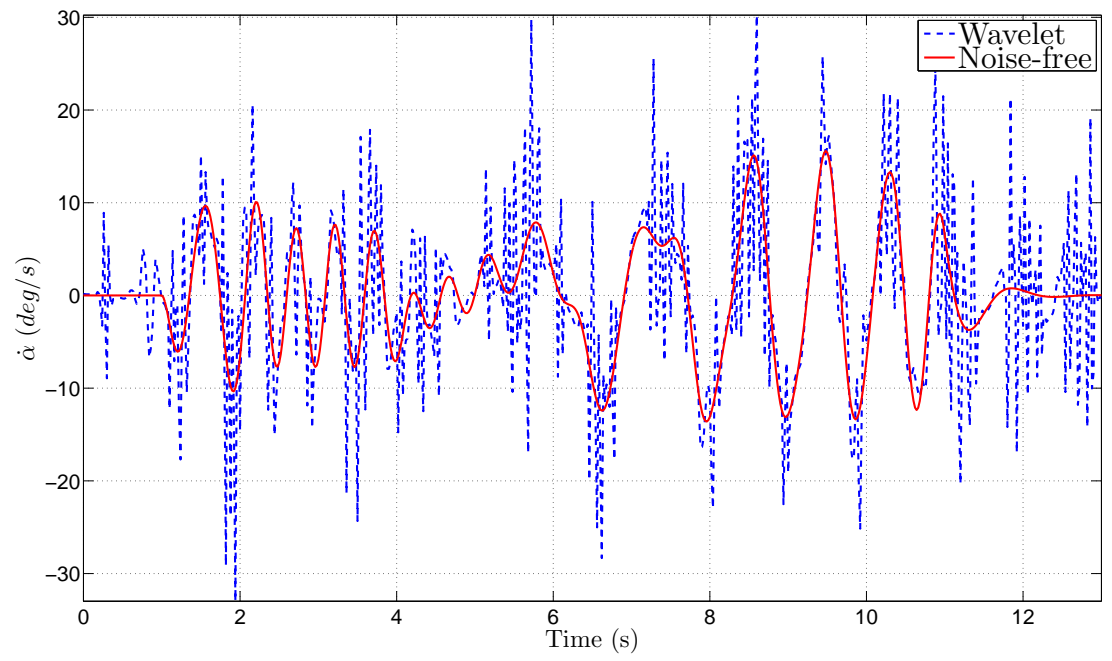

Fig. 1. Example of $\dot{\alpha}$ estimation - White-noise - Wavelet (dashed) and true noise-free signal (solid) 


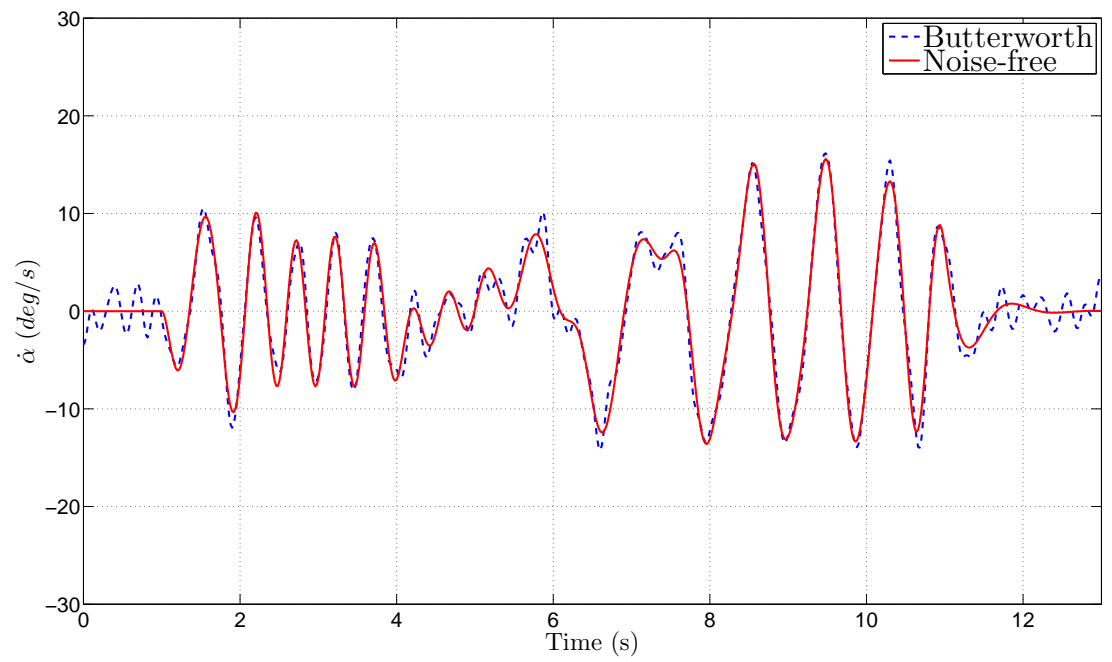

Fig. 2. Example of $\dot{\alpha}$ estimation - White-noise - Butterworth (dashed) and true noise-free signal (solid) 